and R。M.Thaler, Phys. Rev. 109, 2133 (1958)。

These authors treat the potential nonrelativistically; to convert the potential to the conventional relativistic form, as the fourth component of a four-vector, we have multiplied their result by $M_{K} / E_{K}$.
${ }^{8} \mathrm{M}$ 。A. Melkanoff, O。R. Price, D. J. Prowse, D. H. Stork, and $\mathrm{H}$ 。K. Ticho, Proceedings of the International Conference on the Nuclear Optical Model, Florida State University Studies, No. 32 (The Florida State University, Tallahassee, 1959).

\title{
FINITE SELF-ENERGY OF CLASSICAL POINT PARTICLES
}

\author{
R. Arnowitt* \\ Department of Physics, Syracuse University, Syracuse, New York \\ S. Deser* \\ Department of Physics, Brandeis University, Waltham, Massachusetts \\ and \\ C. W. Misner ${ }^{\dagger}$ \\ Palmer Physical Laboratory, Princeton University, Princeton, New Jersey
}

(Received February 10, 1960)

The infinite mass self-energy difficulties of quantum field theory already occur, as is wellknown, in the corresponding classical theories. Although cutoffs may be introduced to effect renormalization in both the classical and quantum cases, such procedures are physically unsatisfactory. We wish to point out in this note that at least for the static (Coulomb-type) contribution, one obtains finite results for the classical selfenergies if the gravitational contribution to the total energy is included. Furthermore, it will be shown rigorously (in the static case) that the natural cutoff furnished by general relativity implies that all the mass of a point charge arises from its total self-field and that a neutral particle has no mass.

It has previously been shown ${ }^{1}$ that the energy of the gravitational field is given $b^{2}$

$$
E=\int\left(g_{i j, j}-g_{j j, i}\right) d S_{i},
$$

where $d S_{i}$ is a two-dimensional surface element at spatial infinity. When point particles or other fields (such as the electromagnetic field) are coupled to the gravitational field, Eq. (1) represents the total energy of the combined system. ${ }^{3}$ We begin by considering the metric field arising from the coupling of a neutral static point particle. In isotropic coordinates $\left[g_{i j}=\chi^{4}(r) \delta_{i j}\right]$ the relevant field equation is

$$
\begin{aligned}
-2\left(R_{0}^{0}-\frac{1}{2} R\right)\left({ }^{3} g\right)^{1 / 2} & \equiv-8 \chi \nabla^{2} \chi \\
& =-T^{0}{ }_{0}\left({ }^{3} g\right)^{1 / 2}=m_{0} \delta^{3}(\overrightarrow{\mathrm{r}}),
\end{aligned}
$$

where $m_{0}$ is the bare mass of the particle and $\delta^{3}(\overrightarrow{\mathrm{r}})$ is invariantly defined in three-space as a scalar density, ${ }^{4}$ i.e., $\int \delta^{3}(\overrightarrow{\mathrm{r}}) d^{3} r=1$. The solution of Eq. (2) which is asymptotically flat is seen to be

$$
\chi(r)=1+m_{0} /[32 \pi r \chi(0)] .
$$

The parameter $m \equiv m_{0} / \chi(0)$ is given in terms of $m_{0}$ by

$$
m=\lim _{\epsilon \rightarrow 0} 2 m_{0}\left[1+\left(1+m_{0} / 8 \pi \epsilon\right)^{1 / 2}\right]^{-1} .
$$

In Eq. (4), $\epsilon$ is essentially the "radius" of the $\delta^{3}$ function. This relation between $m$ and $m_{0}$ is a consequence of explicitly considering the source term in Eq. (2). From Eq. (1) one sees that $E=m$. That this energy is to be correctly interpreted as the total mass of the particle follows from the fact that an isotropic time-sym metric metric possesses no dynamical gravitational modes. ${ }^{5}$ Thus $E$ represents the mass of a gravitationally clothed one-particle system (and no dynamical gravitational excitations). From Eq. (4) then, this total mass approaches zero as $\left(32 \pi m_{0} \epsilon\right)^{1 / 2}$. The gravitational self-mass for a neutral particle therefore cancels the bare mass $m_{0}$. The physical origin of this result $(m=0)$ is connected to the well-known fact that there is an upper limit, in general relativity, to the amount of energy that can reside in a given region. As the size of the particle (here $\epsilon$ ) goes to zero its mechanical energy content must vanish. We may note that the (incorrect) weak-field result for the self-mass could be obtained from Eq. (4) by taking the limit $m_{0}$ small before letting $\epsilon$ tend to zero. Here one would find $m=m_{0}-\frac{1}{2} m_{0}{ }^{2} / \epsilon+O\left(1 / \epsilon^{2}\right)$, the standard linearly infinite Coulomb-type self- 
energy. Higher terms in this perturbation expansion are more and more divergent. The correct result, $m=0$, thus indicates the lack of validity of the perturbation approach.

If a particle is coupled to another field of nonzero range, it may be expected to have nonvanishing total mass due to the interaction with the other field. In flat space, one has, for electromagnetic interactions, the infinite Coulomb selfenergy $\frac{1}{2} e^{2} /(4 \pi \epsilon)$. We shall now show that this infinite result is made finite when gravitational coupling is taken into account. The coupling of the electromagnetic field to the gravitational field is included in the generally covariant electromagnetic Lagrangian,

$$
\mathscr{L}_{\mathrm{em}}=A_{\nu} \mathcal{F}^{\mu \nu}, \mu^{+\frac{1}{4}(-g)^{-1 / 2} \mathfrak{F}^{\mu \nu}{ }_{\mathcal{F}}^{\alpha \beta}} g_{\mu \alpha} g_{\nu \beta} \text {, }
$$

where $A_{\mu}$ is the vector potential and $\mathfrak{F}^{\mu \nu}$ is the field strength tensor density. We are here employing that formulation of the electromagnetic field in which $A_{\mu}$ and $\mathscr{F} \mu \nu$ are to be varied independently. This gives rise to the usual Maxwell equations as well as to the relations between potentials and field strengths. The analysis for finding the independent dynamical variables of the Maxwell field proceeds just as in Lorentz covariant electrodynamics. ${ }^{6}$ One finds that the canonical variables are $A_{i} T$ and $\mathcal{E}^{i T} \equiv \mathcal{F} 0 i T$, the transverse parts of $A_{i}, \mathcal{E}^{i}\left(A_{i}{ }^{T}, i=0=\mathcal{E}^{i T}, i\right)$. The longitudinal part of $\mathcal{E}^{i}$ is determined by the differential constraint equation $\mathcal{E}^{i}, i=\rho$ where $\rho$ is the charge density.

In order to investigate the self-energy of a point charge, one must consider the coupled Einstein-Maxwell equations. For the static case these are

$$
\begin{gathered}
-8 \chi \nabla^{2} \chi=m_{0} \delta^{3}(\overrightarrow{\mathrm{r}})+\frac{1}{2} \chi^{-2} \mathcal{E}^{i} \mathcal{E}^{i} \\
\mathcal{E}_{, i}^{i}=e \delta^{3}(\overrightarrow{\mathrm{r}})
\end{gathered}
$$

The term $\frac{1}{2} \chi^{-2} \mathcal{E}^{i} i_{\mathcal{E}}$ in Eq. (6a) is the electromagnetic energy density. The solution for $\chi(r)$ is

$$
\chi^{2}(r)=(1+m / 32 \pi r)^{2}-(e / 16 \pi r)^{2},
$$

where $m$ is related to the particle's bare mass $m_{0}$ and its charge $e$ by

$$
m=\lim _{\epsilon \rightarrow 0} 16 \pi\left\{-\epsilon+\left[\epsilon^{2}+(e / 8 \pi)^{2}+m_{0} \epsilon / 8 \pi\right]^{1 / 2}\right\} .
$$

From Eq. (1), we again have $E=m$. As in the neutral case there are no dynamical gravitational modes excited, nor are there any transverse canonical electromagnetic modes present since the solution of Eq. (6b) we have taken, $\vec{E}=(e / 4 \pi) \vec{\nabla} r^{-1}$, is purely longitudinal. Thus $m$ is simply the total mass of the charged particle. The mass is finite and equals $2|e|$, or in conventional units,

$$
m=\gamma^{-1 / 2}|e| /(4 \pi)^{1 / 2} \text {. }
$$

It should be stressed that this finite result has been obtained entirely within the framework of standard Einstein-Maxwell theory. The possibility of a finite combined gravitational-electrostatic self-energy can be seen already in Newtonian physics where $-\frac{1}{2} \gamma \mathrm{m}^{2} / \epsilon$ would compensate $e^{2} / 4 \pi \epsilon$ if Eq. (9) held. Of course, the relation (9) between $m$ and $e$ was not chosen just to achieve this compensation, but was forced in order that solutions of the general relativistic Eqs. (6) exist. That the total mass here is not zero, unlike the neutral case, arises from the fact that the electric energy term in Eq. (6a) is spread out in a nonzero region. Similarly, an electrically uncharged particle coupled to a Yukawa field, for example, will possess a nonvanishing finite self-mass independent of $m_{0}$. As in the neutral case, the weak-field limit can be obtained by expanding in $e$ and $m_{0}$ before letting $\epsilon \rightarrow 0$. One would get then $m=m_{0}-\frac{1}{2} \gamma m_{0}^{2} / \epsilon$ $+\frac{1}{2} e^{2} / 4 \pi \epsilon+O\left(1 / \epsilon^{2}\right)$, and no compensation occurs since $e$ and $m_{0}$ are independent parameters, which shows again the lack of validity of the perturbation expansion.

The solutions derived here for the neutral and charged one-particle states differ from the conventional Schwarzschild and Reissner-Nordstrom metrics in two ways. First, these usual metrics can be seen not to satisfy Eqs. (2) and (6), respectively, for any finite mass $m_{0}$ and in fact only solve the field equations for $r>0$. Hence the correct relation between the parameter $m$ and the bare mass $m_{0}$ and charge $e$ cannot be made for these solutions, i.e., one would fail to discover that, for a point particle, $m$ is determined by $e$. Second, the way in which the metric enters into the matter stress tensor is uniquely determined by the canonical formalism; this is not the case for the usual discussion of coupling in which there is still ambiguity. ${ }^{7}$

The extension of the above results for the mass of a single point source to two-body solutions is (aside from its intrinsic interest) necessary to see whether these masses enter properly into the interaction energy. We have been able to carry out the analysis for the case of two charges of the same sign. For simplicity, we consider here the situation of equal bare masses and equal 
charges. In Eqs. (6), the term $m_{0} \delta^{3}(\overrightarrow{\mathrm{r}})$ is replaced by $m_{0}\left[\delta^{3}\left(\overrightarrow{\mathrm{r}}-\overrightarrow{\mathrm{r}}_{1}\right)+\delta^{3}\left(\overrightarrow{\mathrm{r}}-\overrightarrow{\mathrm{r}}_{2}\right)\right]$ and $e \delta^{3}(\overrightarrow{\mathrm{r}})$ by $e\left[\delta^{3}\left(\overrightarrow{\mathbf{r}}-\overrightarrow{\mathbf{r}}_{1}\right)+\delta^{3}\left(\overrightarrow{\mathbf{r}}-\overrightarrow{\mathbf{r}}_{2}\right)\right]$, where $\overrightarrow{\mathbf{r}}_{1}$ and $\overrightarrow{\mathbf{r}}_{2}$ are the positions of the point charges. The solution corresponding to Eq. (7) generalizes to the form ${ }^{8}$ $\chi^{2}(\overrightarrow{\mathrm{r}})=\psi^{2}(\overrightarrow{\mathrm{r}})-\varphi^{2}(\overrightarrow{\mathrm{r}})$ where $\psi=1+(a / 32 \pi)\left(\left|\overrightarrow{\mathrm{r}}-\overrightarrow{\mathrm{r}}_{1}\right|^{-1}\right.$ $\left.+\left|\overrightarrow{\mathbf{r}}-\overrightarrow{\mathbf{r}}_{2}\right|^{-1}\right)$ and $\varphi=(e / 16 \pi)\left(\left|\overrightarrow{\mathbf{r}}-\overrightarrow{\mathbf{r}}_{1}\right|^{-1}+\left|\overrightarrow{\mathbf{r}}-\overrightarrow{\mathrm{r}}_{2}\right|^{-1}\right)$. Here the total energy $E=2 a$ and the field equations give

$$
\begin{aligned}
& E=32 \pi\{-\epsilon+ {\left[\epsilon^{2}+(e / 8 \pi)^{2}(1+x)^{2}\right.} \\
&\left.\left.+\left(m_{0} \epsilon / 8 \pi\right)(1+x)\right]^{1 / 2}\right\}[1+x]^{-1},
\end{aligned}
$$

where $x=\epsilon /\left|\overrightarrow{\mathbf{r}}_{1}-\overrightarrow{\mathbf{r}}_{2}\right|$. In the limit $\epsilon=0$, one obtains $E=4|e|=2 m$. Since the dynamical modes of both the Einstein and Maxwell fields again vanish, $E$ here represents the total energy of the two particles. For two neutral particles $(e=0)$, one has $E=0$ in accord with the one-particle result. When $e \neq 0$, one would have expected for large $r_{12} \equiv\left|\overrightarrow{\mathbf{r}}_{1}-\overrightarrow{\mathrm{r}}_{2}\right|$ that $E-2 m=\left[\left(e^{2} / 4 \pi\right)-\gamma m^{2}\right] / r_{12}$. However, this interaction energy vanishes on account of Eq. (9). The rigorous result above $(E=2 m)$ shows that the cancellation between the electrostatic and gravitational interaction energies holds ${ }^{9}$ even for small $r_{12}$. While these results are consistent with those for one body, it would be of interest to examine the case of opposite charges where the cancellation would not be expected. This case cannot be obtained merely by changing the sign of the $\left|\overrightarrow{\mathbf{r}}-\overrightarrow{\mathbf{r}}_{2}\right|^{-1}$ term in $\varphi(\overrightarrow{\mathbf{r}})$, since the electric field $\overrightarrow{\mathcal{E}}$ corresponding to such a metric has a transverse part, $\overrightarrow{\mathcal{E}} T$, so that the total energy now includes a contribution from these pure electric dynamical modes. We have not been able to solve Eq. (6a) with $\overrightarrow{\mathcal{E}}=e \nabla\left(\left|\overrightarrow{\mathrm{r}}-\overrightarrow{\mathrm{r}}_{1}\right|^{-1}\right.$ $\left.-\left|\overrightarrow{\mathbf{r}}-\overrightarrow{\mathrm{r}}_{2}\right|^{-1}\right)$ for an asymptotically flat $g_{i j}$.

The compensation of the usual infinite static self-energies obtained here arises due to the distinctive nature of the gravitational coupling which modifies the free Lagrangians of all fields rather than introducing an additive interaction Lagrangian. Ultimately, the interest of these classical results rests on their extension to quantum theory. Of course, the criteria for the usefulness of a gravitational cutoff in that domain would depend not merely on finiteness but on the numerical values it would yield.
* Supported in part by a National Science Foundation Research Grant.

${ }^{\dagger}$ Alfred P. Sloan Research Fellow.

${ }^{1}$ R. Arnowitt, S. Deser, and C. W. Misner, Nuovo cimento (to be published); Phys. Rev。116, 1322 (1959); Phys. Rev. (to be published); Phys. Rev. (to be published); which will be referred to as I, II, III, and IV, respectively.

${ }^{2}$ We use units such that $\kappa=16 \pi \gamma c^{-4}=c=1$, where $\gamma$ is the Newtonian gravitational constant. Latin indices run from 1 to 3 , Greek from 0 to 3 . The summation convention holds even if repeated indices are both covariant. A comma represents ordinary differentiation. It should be mentioned that the energy of Eq. (1) depends only on the spatial part of the metric which constitutes the initial Cauchy data. Thus, to calculate the energy of the system one does not have to know $g_{00}$ or any time development of the system. The same expression for the energy may also be obtained from the usual surface integral forms of the energy [see for example L. Landau and E. Lifshitz, Classical Theory of Fields (Addison-Wesley Publishing Company, Inc., Reading, Massachusetts, 1951), Eq.(11-88), where $g_{0 \mu}$ cancels out in the linearized asymptotic form valid at spatial infinity.

${ }^{3}$ That this is reasonable follows from the fact that the energy of a system is the coefficient of $1 / v$ in the asymptotic expansion of $g i j$; see also IV.

${ }^{4}$ The $\left({ }^{3} g\right)^{1 / 2}$ factor on the left-hand side of Eq. (2) is uniquely determined when one puts the Lagrangian including matter variables into canonical form (see IV). That it must be present follows obviously from the scalar density nature of the $\delta^{3}(r)$ function.

${ }^{5}$ If one chooses the coordinate conditions $x^{i}=2 g_{i}$ $-\left(1 / 2 \nabla^{2}\right) g^{T}, i, t=-\left(1 / 2 \nabla^{2}\right)\left(\pi T^{T}+\nabla^{2} L^{L}\right)$ (in the notation of reference 1 ), the analysis of the Nuovo cimento Letter would show that the canonical variables are $g_{i j} T T$ and ${ }_{\pi} i j T T$. These dynamical modes vanish everywhere when the spatial metric is isotropic and $\pi i j=0$ (initially static situation)。

${ }^{6} \mathrm{R}$. Arnowitt and S。Deser, Phys. Rev。 113,745 (1959).

${ }^{7}$ Thus, in the usual treatment of the interior Schwarzschild solution, the bare rest-mass density is never even introduced. The standard "proper rest-mass density" parameter includes clothing effects in its definition in an unanalyzed manner.

${ }^{8}$ See C. W. Misner and J.A. Wheeler, Ann. Phys. 2, 594 (1957).

${ }^{9} \mathrm{~A}$ corroboration of the absence of interaction energy and therefore of forces between the particles is furnished by the fact that in the case $m=2|e|$, the initial value metric described by $\psi$ and $\varphi$ determines a static solution of the Einstein equations [A. Papapetrou, Proc. Roy. Irish Acad. A51, 191 (1947)]. 